\title{
Brown Dwarf Companions to White Dwarfs
}

\author{
Matt R. Burleigh*, Paul R. Steele ${ }^{\dagger}$, Paul D. Dobbie**, Jay Farihi*, Ralf \\ Napiwotzki ${ }^{\ddagger}$, Pierre F. L. Maxted ${ }^{\S}$, Martin A. Barstow*, Richard F. \\ Jameson*, Sarah L. Casewell*, Boris T. Gaensicke ${ }^{\mathbb{I}}$ and Tom R. Marsh ${ }^{\mathbb{I}}$ \\ ${ }^{*}$ Dept. of Physics and Astronomy, University of Leicester, Leicester, LE1 7RH, UK \\ ${ }^{\dagger}$ Max-Planck-Institut für extraterrestrische Physik, Giessenbachstraße, 85748, Garching, Germany \\ ${ }^{* *}$ Australian Astronomical Observatory, PO Box 296, Epping, Sydney, NSW 1710, Australia \\ ${ }^{\ddagger}$ Centre for Astrophysics Research, University of Hertfordshire, Hatfield AL10 9AB, UK \\ ${ }^{\S}$ Astrophysics Group, Keele University, Staffordshire, ST5 5BG, UK \\ ${ }^{I I}$ Department of Physics, University of Warwick, Coventry, CV4 7AL, UK
}

\begin{abstract}
Brown dwarf companions to white dwarfs are rare, but recent infra-red surveys are slowly revealing examples. We present new observations of the post-common envelope binary WD0137-349, which reveals the effects of irradiation on the $\approx 0.05 M_{\odot}$ secondary, and new observations of GD 1400 which show that it too is a close, post-common envelope system. We also present the latest results in a near-infrared photometric search for unresolved ultra-cool companions and to white dwarfs with UKIDSS. Twenty five DA white dwarfs were identified as having photometric excesses indicative of a low mass companion, with 8-10 of these having a predicted mass in the range associated with brown dwarfs. The results of this survey show that the unresolved $(<2$ ") brown dwarf companion fraction to DA white dwarfs is $0.3 \leq f_{\mathrm{WD}+\mathrm{BD}} \leq 1.3 \%$.
\end{abstract}

Keywords: White Dwarfs, Brown Dwarfs, Binaries

PACS: 97,98

\section{INTRODUCTION}

Large-scale near-infrared (NIR) surveys of white dwarfs (WDs) are ideal for the searches and studies of substellar companions. A typical WD is $10^{3}-10^{4}$ times fainter than its main sequence (MS) progenitor, significantly reducing the brightness contrast problem when searching for cool, low mass secondaries. In addition, the spectral energy distributions of WDs (blue) and their low mass companions (red) are markedly different, facilitating easy separation of the components in broadband photometry and enabling straightforward spectroscopic follow-up (e.g. Dobbie et al. 7).

Searches for substellar companions to WDs allows for the investigation of the known deficit of brown dwarf (BD) companions to MS stars [22, 14]. Since the ages of WDs can be relatively well constrained, any $\mathrm{BD}$ companions can potentially be regarded as "benchmarks" for testing evolutionary and atmospheric models [26]. The closest $\mathrm{WD}+\mathrm{BD}$ binaries might also represent either a channel for cataclysmic variable $(\mathrm{CV})$ evolution or the end state of CV evolution, in which the secondary has become highly evolved through mass transfer [25]. Indeed, there are a growing number of CVs with confirmed BD secondaries (e.g. Littlefair et al. 19, 20). In close detached binaries, the BD is expected to be irradiated by the WD's high UV flux, possibly leading to substantial temperature differences between the "day" and "night" hemispheres. Such systems 
can provide laboratories for testing models of irradiated "hot Jupiter" atmospheres (e.g. HD 189733b; Knutson et al. 17). However, detached BD companions to WDs are rare. [10] calculate a binary fraction of $f_{\mathrm{WD}+\mathrm{dL}}<0.5 \%$ for L dwarfs.

Radial velocity and proper motion surveys, and searches for NIR excesses have so far found only five confirmed examples: GD 165 (DA+dL4, Becklin and Zuckerman 1), GD 1400 (DA+dL6 - 7; Farihi and Christopher 11, Dobbie et al. 7), WD 0137 - 349 (DA+dL8; Maxted et al. 21, Burleigh et al. 2), PHL 5038 (DA+dL8; Steele et al. 27), and the first T dwarf + WD pair, LPSM 1459+0857 AB [6]. GD 165, PHL 5038 and LPSM $1459+0857 \mathrm{AB}$ are wide systems with projected separations of $120 \mathrm{AU}, 55 \mathrm{AU}$ and $\sim 20,000$ AU respectively, whereas GD 1400 and WD 0137-349 are in much closer orbits with periods of $\approx 10$ hours (see below) and 116 mins respectively.

\section{NEW OBSERVATIONS OF THE CLOSE BINARIES WD 0137-349 AND GD 1400}
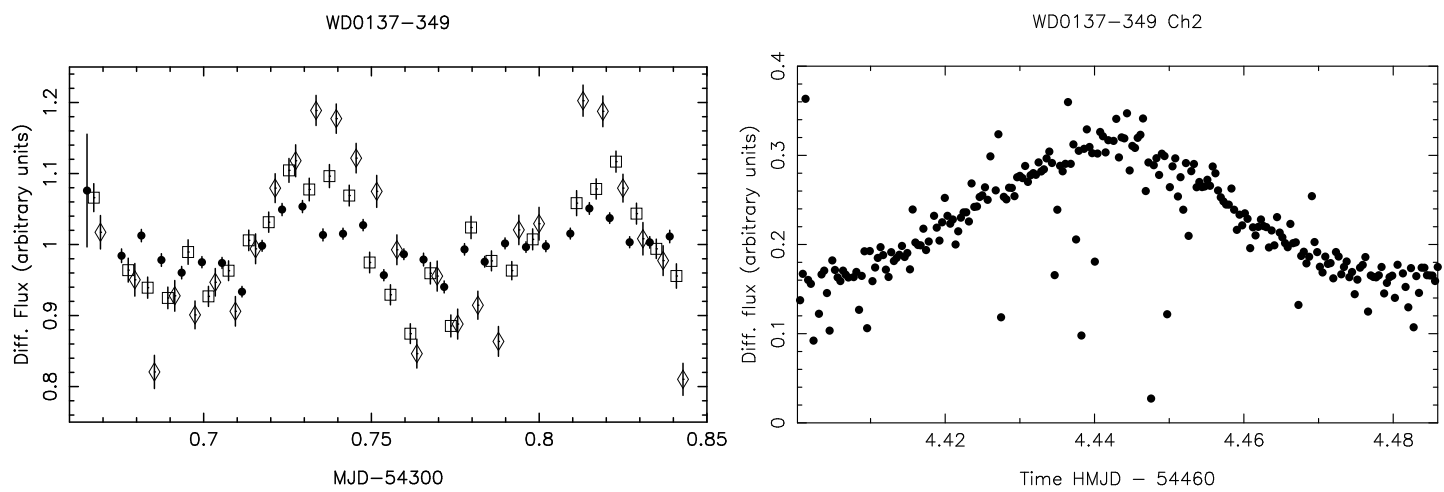

FIGURE 1. Right: Near-IR light curves of WD0137-349 obtained with IRIS2 on the AAT. Filled circles: $J$-band, open squares: $H$-band, open diamonds: $K$-band. Left: Mid-IR, Spitzer $4.5 \mu$ m light curve.

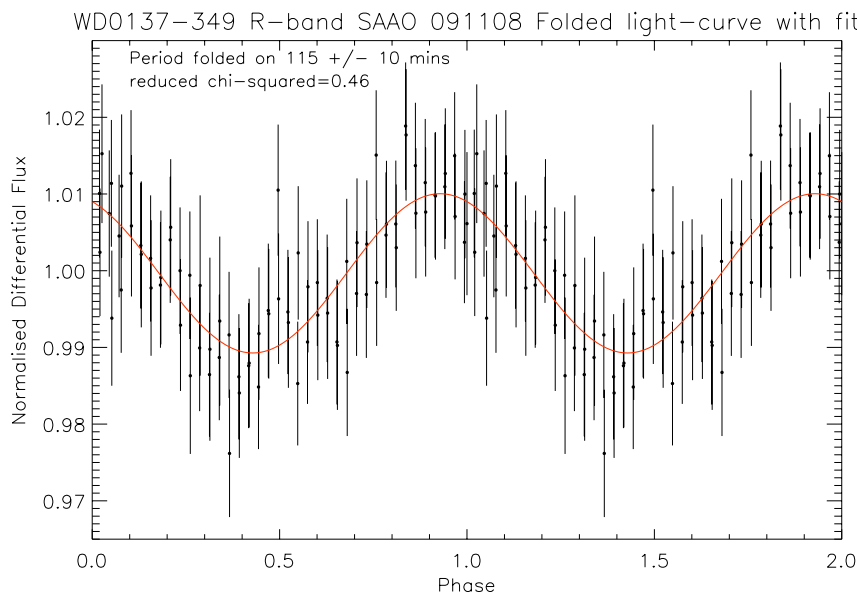

FIGURE 2. Optical $R$-band light curve of WD0137-349 obtained with the SAAO 1.0m. 
We have continued to observe WD 0137-349 since its discovery by [21]. Multiwaveband light curves from the optical to the mid-infrared show that the BD is being heated on the hemisphere facing the WD, producing significant variability on the 116 min orbital period, from $\pm 10 \%$ at $4.5 \mu \mathrm{m}$ to $\pm 1 \%$ at $R$ (Figure 1and Figure 2). The optical modulations are a slightly surprising discovery, and we are modeling these light curves to further investigate the effects of irradiation on the substellar component's atmosphere.

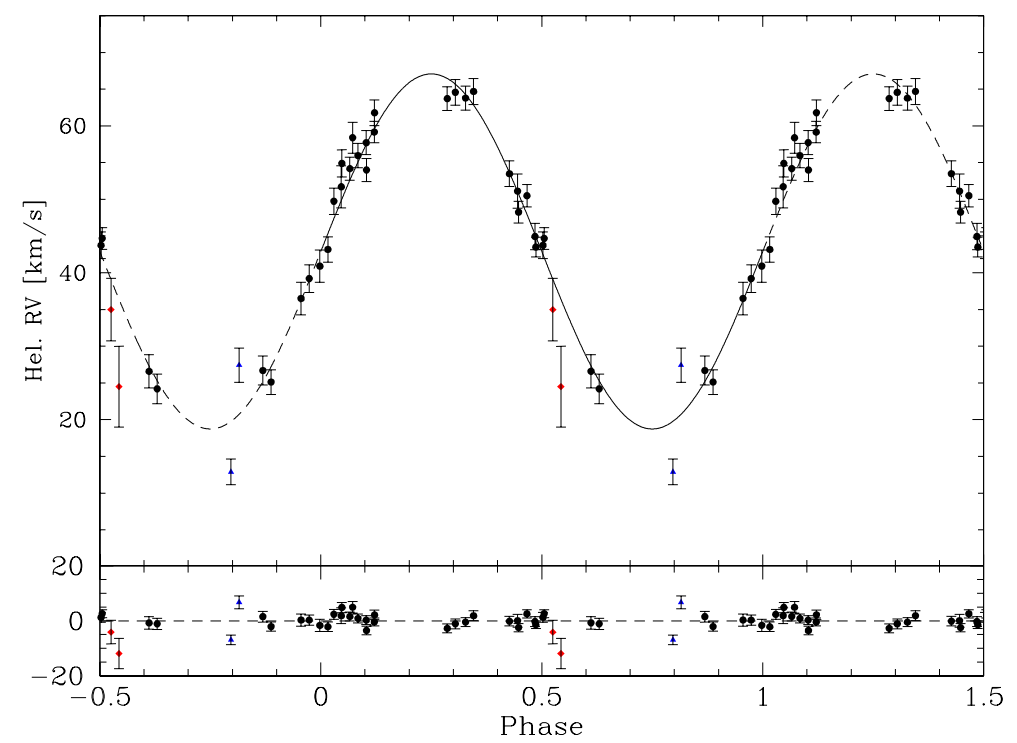

FIGURE 3. Radial velocity measurements of the white dwarf component of GD1400, obtained with UVES on the VLT, folded on the best-fit period of 9.98 hours.

Radial velocity observations of GD 1400 obtained with UVES on the VLT (Burleigh et al. 2011, in prep.) conclusively show that it is a close, post-common envelope binary with an orbital period $P_{\text {orb }}=9.98 \mathrm{hrs}$ (Figure 3). Although $\mathrm{H}$ emission from the brown dwarf is tentatively detected at one phase (possibly due to a flare rather than irradiation), Burleigh et al. are unable to determine its radial velocity. From the mass function they place a lower limit on the BD mass $M_{2} \approx 0.06 M_{\odot}$, with a lower limit on the WD mass $M_{2} \approx 0.55 M_{\odot}$.

Near-IR photometry of GD 1400, obtained sporadically across the $9.98 \mathrm{hr}$ orbital period by Burleigh et al. (2011, in prep.), shows no variability. This is perhaps unsurprising: the greater separation of the two components compared to WD0137-349, and the cooler WD primary $\left(T_{\mathrm{eff}} \approx 11,500 \mathrm{~K}\right.$ versus $T_{\mathrm{eff}} \approx 16,000 \mathrm{~K}$ for WD0137-349) suggests much weaker irradiation of the BD secondary.

GD 1400B is the second substellar object that must have survived engulfment by its parent star's atmosphere during one of the giant phases of stellar evolution, after WD 0137 - 349. From consideration of the white dwarf masses, it is likely that GD 1400 underwent common envelope evolution on the asymptotic giant branch and that the brown dwarf originally orbited the main sequence progenitor star between 0.1 and 1 AU, while WD 0137 - 349 went through a common envelope stage on the red giant branch and had an original orbital separation $\lesssim 0.1 \mathrm{AU}$. These binaries are the direct 

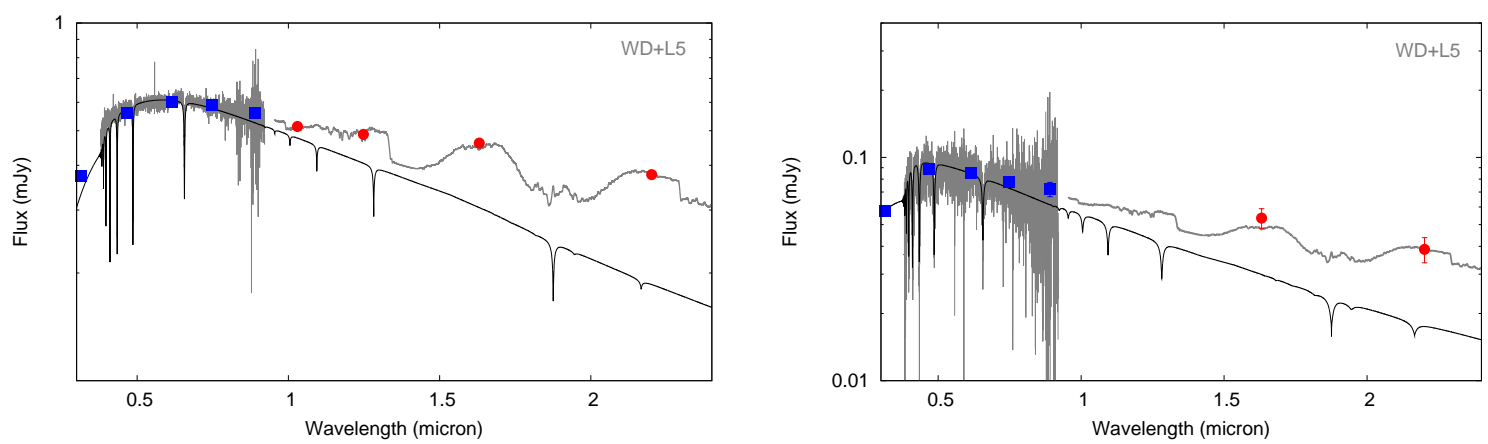

FIGURE 4. Two examples of DA WDs that show a NIR excess. SDSS photometry (squares), available UKIDSS photometry (circles), a model atmospheric spectrum (black solid), the SDSS spectrum (light grey) and a composite WD + companion model (as labeled) are plotted for each.

descendants of intermediate-mass giant stars with brown dwarf companions in orbits $a \sim$ few AU.

The WD component of GD 1400 is also a ZZ Ceti pulsator. [12] identified three significant frequency components at $823.2 \mathrm{~s}, 727.9 \mathrm{~s}$ and $462.2 \mathrm{~s}$, the latter having the strongest amplitude.

\section{A SURVEY FOR WD+BD BINARIES WITH UKIDSS}

New large infra-red sky surveys will reveal more examples of WD+BD binaries, and allow us to better constrain their frequency. Here, we present preliminary results from the UKIRT Infrared Deep Sky Survey (UKIDSS, Lawrence et al. 18, Warren et al. 29). UKIDSS is the near-IR counterpart of the Sloan Digital Sky Survey and is several magnitudes deeper than the previous 2MASS survey, although unlike the latter UKIDSS will cover only $\sim 20 \%$ of the sky. UKIDSS uses the $0.21 \mathrm{deg}^{2}$ field of view Wide Field Camera [4] on the 3.8m UKIRT telescope on Mauna Kea, Hawaii. The UKIDSS surveys began in May 2005 and are expected to be completed in 2012. The survey of most relevance to this work is the Large Area Survey (LAS), which aims to cover $\sim 4000 \mathrm{deg}^{2}$ of the Northern Sky coincident with the SDSS. The LAS makes observations in the $Y J H K$ filters to a $5 \sigma$ depth for point sources of $Y \approx 20.2, J \approx 19.6, H \approx 18.9$ and $K \approx 18.2$ [15].

\section{Candidate selection}

Isolated WDs and candidate binaries were identified using the methods described in Steele et al. (2011, in prep.). For UKIDSS DR8 the cross correlation produced 3109 exclusive matches with the SDSS DR4 catalogue [9], and 163 with the McCook \& Sion catalogue (MS99, McCook and Sion 23). 
In brief, we have used Pierre Bergeron's synthetic colours and evolutionary sequences for DA WDs ${ }^{1}$. The model grid extends from $1500<T_{\mathrm{eff}}<100,000 \mathrm{~K}$ and $7.0<\log g<9.0$ but was linearly extrapolated to $6.5<\log g<9.5$ to include the lower and higher mass WDs, but to preclude any possible sub-dwarf contamination of the sample. This provided theoretical absolute magnitudes for the SDSS $i^{\prime}$ and the 2MASS $J H K_{\mathrm{S}}$ filters, as well as an estimated distance to the WD and cooling age of the system. The 2MASS magnitudes were converted to the corresponding UKIDSS filters using the colour transformations of [3]. Out of the 2675 WDs classified by EIS06 and MS99 as DA WDs, 1040 had both $H$ and $K$-band photometry and fell within the extended model grid.

We identified candidate stars as those showing at least a $>3 \sigma$ excess in the UKIDSS $H$ and $K$-bands (or $K$-band only) when compared to the model predicted values. 314 such excesses were identified, with 275 of these accounted for as previously identified binaries from an optical excess in SDSS (or other optical data). This left 39 candidate WDs with an apparent NIR excess. Ten of these appear to be caused by foreground or background contamination (e.g. due to a nearby red star or galaxy), 25 by a previously unidentified companion and 4 by a debris dust disk.

Empirical models for low mass sub-stellar objects were then added to a WD synthetic spectrum and these composites were compared to UKIDSS photometry to obtain an approximate spectral type for the putative companion. Due to errors in temperature, surface gravity and therefore distance, estimates of the companion spectral types are likely within \pm 1 spectral types of the best fitting composite WD + companion model spectrum. The results break down as follows; $1 \mathrm{dM} 3,2 \mathrm{dM} 5,3 \mathrm{dM} 6,5 \mathrm{dM} 7,2 \mathrm{dM} 8,1$ dM9, 2 dL0, 2 dL1, 1 dL4, 3 dL5, 1 dL7, 1 dL8 and 1 dT3.

\section{Low Mass Star or Brown Dwarf?}

In order to assess whether or not each putative companion is a low mass MS star or a $\mathrm{BD}$ an estimate of the mass of the secondary has been calculated. Firstly an age of the WD was calculated by the addition of the predicted WD cooling age and MS lifetime of the progenitor star. This was estimated using the initial-final mass relationship of [8], which is valid for initial masses $>1.6 M_{\odot}[16]$. An approximate MS lifetime can then be calculated from the models of [13]. It should be noted that for WDs where $M_{\mathrm{WD}}<0.5 M_{\odot}$ it is highly likely that the star has evolved through mass transfer and for these stars an age can not be calculated through this method. However, this can be seen as further evidence for the existence of a secondary star. For these stars, a lower limit on the mass of the secondary is estimated by using the cooling age of the WD.

We then estimate a mass for the secondary by interpolating the Lyon Group atmospheric models (e.g. Chabrier et al. 5), given the age of the WD and an estimate of effective temperature of the companion. We estimated these temperatures by comparison with observed M, L and T dwarfs (28) and assuming an error of \pm 1 spectral type.

1 http: //www.astro.umontreal.ca/ bergeron/CoolingModels/ 


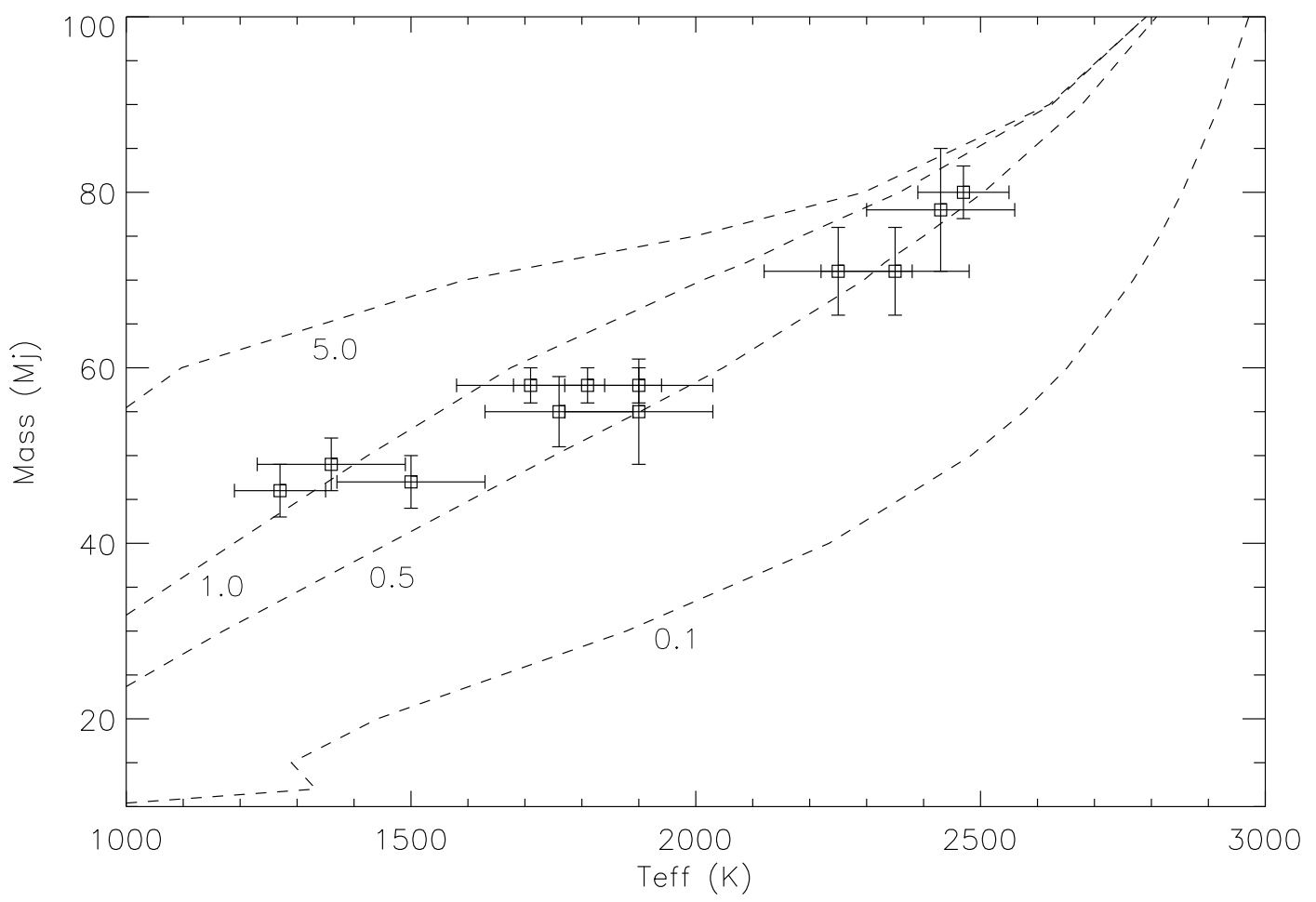

FIGURE 5. Predicted masses of companions below $80 M_{\text {Jup }}$ based on the Lyon Group models. The dashed lines indicate constant age in Gyr.

The results are plotted in Figure 5.

If we assume a value of $70 M_{\text {Jup }}$ as a conservative cut-off mass for the stellar-substellar boundary, then 8-10 new DA WD+BD binaries binaries have potentially been discovered (1 DA+L8 has been spectroscopically confirmed; Steele et al. 27).

\section{Limits on Unresolved Ultra-Cool Companions to DA WDs}

Atmospheric modeling successfully fitted 538 DA WDs to within $3 \sigma$ of both their UKIDSS $H$ and $K$-band photometry. We will use these stars to estimate the limits on the spectral types of unresolved companions to DA WDs and hence determine the sensitivity of UKIDSS to both L and T dwarfs in WD binaries. The method used to calculate these limits is detailed below.

In order to place a limit on the spectral type of the coolest detectable unresolved companion to each DA WD we required the absolute magnitudes for the spectral types of low mass objects ranging from dL0-dT8. [24] lists known M, L and T dwarfs with 2MASS $J H K_{\mathrm{S}}$ photometry ranging from spectral types dM5-dT8 and also has measured parallaxes for many of these. We converted the 2MASS $J H K_{\mathrm{S}}$ photometry for each object to the UKIDSS $J H K$ photometric system using the colour corrections of [3]. 

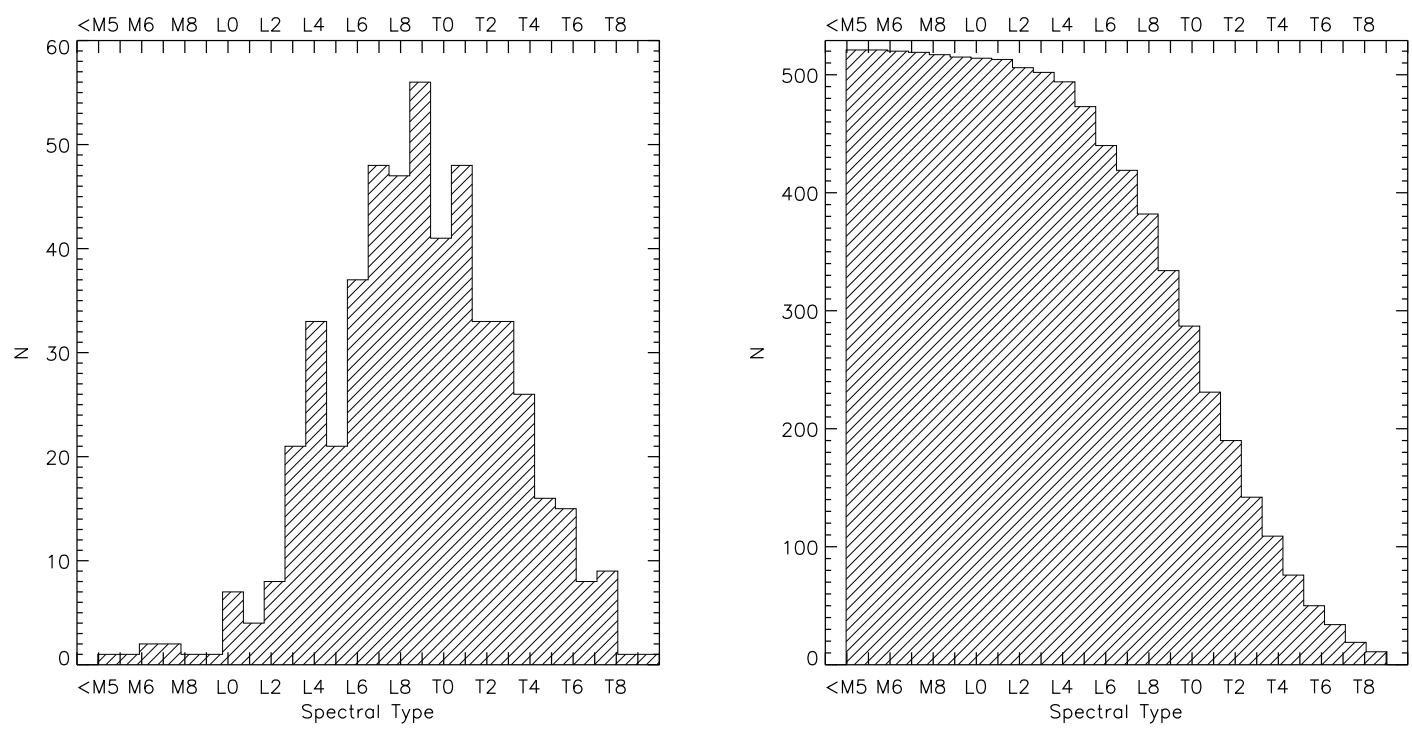

FIGURE 6. Left to right: (1) Distribution of limits on unresolved companions to the "single" DA white dwarfs (with well determined parameters) detected in UKIDSS DR8. (2) Same as (1) but plotted as a cumulative histogram.

Taking only those with a measured parallax, and thus an estimated distance, the $K$-band photometry was scaled to $10 \mathrm{pc}$ for each spectral type to obtain an estimated absolute $K$ band magnitude. For multiple stars of the same spectral type we used an average of the magnitudes. For the few spectral types that had no parallax measurement we performed a linear interpolation to predict an absolute magnitude.

For each apparently single DA WD we predicted the expected $K$-band magnitude by folding the WD's atmospheric model through the UKIDSS $K$-band filter transmission profile. The observed $K$-band magnitude was not utilised, as any of the WDs could be in excess within the observed errors. This would have resulted in an over-estimate of the limiting spectral type for the companion to these stars. We then calculated a $3 \sigma$ detection limit for each WD by using the observed errors listed in the UKIDSS DR8, and adding these to the predicted magnitudes. The distance to each WD was calculated using the Bergeron cooling models using the effective temperatures and surface gravities produced from the automated fit of EIS06. For isolated DA WDs only found in MS99, temperature estimates were obtained from the literature or an automated fit to the stars' optical photometry. For stars without a measured surface gravity, we assumed a $\log g=8.0$. This applied to only 11 WDs in the sample, which is not a large enough number to create a distance bias that could later affect the statistics. Subsequently, the grid of M, $\mathrm{L}$ and $\mathrm{T}$ dwarf absolute magnitudes were scaled to the estimated distance of each WD and added to the predicted WD $K$-band magnitudes until a match was made with the $3 \sigma$ detection limit. The spectral types of all the companion limits were summed and plotted as a histogram and a cumulative histogram in Figure 6. 


\section{Binary Statistics for WDs with Unresolved Substellar Companions}

In order to determine the fraction of WDs with BD companions, we must first estimate the spectral type where the secondary becomes truly substellar. This depends on the age of the secondary, which can be estimated using the total age (MS lifetime + cooling age) of the WD primary. The average age of the WD sample is $1.8 \pm 0.7 \mathrm{Gyr}$. After $\approx 0.1 \mathrm{Gyr}$ BDs cool very slowly, so the average sample age can be used to estimate a spectral type where the stellar/substellar borderline occurs. Taking the upper limit on BD mass as $70 M_{\text {Jup }}$, then an upper limit for the BD spectral type can be taken as L4 (Figure 5).

In order to calculate the $\mathrm{WD}+\mathrm{BD}$ binary fraction, we are going to determine the effective number of detections. This value represents the number of each spectral type detected if the survey were $100 \%$ efficient at detecting companions of all spectral types. The effective number of detections can then be calculated by dividing the actual number of detections by the sensitivity to each spectral type. For example, if the survey sensitivity to companions of spectral types earlier or equal to L0 was 50\%, and $1 \mathrm{LO}$ was detected, then the effective number of detections is 2 . This is then summed for all spectral types to give the total effective number of BDs detected. The BD companion fraction is then the effective number of detections divided by the sample total. An error can be estimated by taking the square root of the reciprocal number of detections. Thus, an upper limit to the fraction of DA WDs with unresolved BD companions is $f_{\mathrm{WD}+\mathrm{dL}} \leq 1.0 \pm 0.3 \%$. A lower limit can be estimated by assuming only 3 candidates ( 2 previously spectroscopically confirmed and 1 proper motion candidate) are real. This gives a final range of $0.3 \leq f_{\mathrm{WD}+\mathrm{dL}} \leq 1.3 \%$.

Although these statistics are not particularly robust, they are suitable as a first approximation using the small numbers available. In order to improve upon these numbers, the sample needs to be enlarged. In the first instance, this will be done by UKIDSS, which is set to be completed by 2012. Looking ahead, future infrared surveys with VISTA and WISE will significantly add to the WD + ultra-cool companion sample.

\section{ACKNOWLEDGMENTS}

PRS is supported by RoPACS, a Marie Curie Initial Training Network funded by the European Commission's Seventh Framework Programme.

\section{REFERENCES}

1. E. E. Becklin, and B. Zuckerman, Nature 336, 656-658 (1988).

2. M. R. Burleigh, et al., MNRAS 373, L55 (2006).

3. J. M. Carpenter, $A J$ 121, 2851-2871 (2001).

4. M. Casali, et al., $A \& A$ 467, 777 (2007).

5. G. Chabrier, et al., ApJ 542, 464 (2000).

6. A. C. Day-Jones, et al., MNRAS in press (2010), arXiv: 1008.2960.

7. P. D. Dobbie, et al., MNRAS 357, 1049 (2005).

8. P. D. Dobbie, et al., MNRAS 369, 383 (2006).

9. D. J. Eisenstein, et al., ApJS 167, 40 (2006).

10. J. Farihi, et al., ApJS 161, 394 (2005). 
11. J. Farihi, and M. Christopher, $A J$ 128, 1868 (2004).

12. G. Fontaine, et al., ApJ 591, 1184 (2003).

13. L. Girardi, et al., $A \& A S$ 141, 371 (2000).

14. D. Grether, and C. H. Lineweaver, ApJ 640, 1051-1062 (2006).

15. P. C. Hewett, et al., MNRAS 367, 454 (2006).

16. J. S. Kalirai, et al., ApJ 676, 594 (2008).

17. H. A. Knutson, et al., Nature 447, 183 (2007).

18. A. Lawrence, et al., MNRAS 379, 1599 (2007).

19. S. P. Littlefair, et al., Science 314, 1578 (2006).

20. S. P. Littlefair, et al., MNRAS 381, 827 (2007).

21. P. F. L. Maxted, et al., Nature 442, 543 (2006).

22. C. McCarthy, and B. Zuckerman, AJ 127, 2871 (2004).

23. G. P. McCook, and E. M. Sion, ApJS 121, 1 (1999).

24. B. M. Patten, et al., ApJ 651, 502 (2006).

25. J. Patterson, et al., PASP 117, 427 (2005).

26. D. J. Pinfield, et al., MNRAS 368, 1281 (2006).

27. P. R. Steele, et al., $A \& A$ 500, 1207 (2009).

28. F. J. Vrba, et al., $A J$ 127, 2948 (2004).

29. S. J. Warren, et al., MNRAS 375, 213 (2007). 